\title{
Histology and histochemistry of the reproductive potential of Acartia clausi (copepoda: calanoida)
}

\author{
F. Morgado*, S. Terdalkar*, J.R. Gadelha*,*** and M.L. Pereira**
}

*CESAM \& Departamento de Biologia, Universidade de Aveiro, Campus Universitário de Santiago, 3810 193 Aveiro, Portugal

**Departamento de Biologia \& CICECO, Universidade de Aveiro, Campus Universitário de Santiago, 3810-193 Aveiro, Portugal

***CAPES Foundation, Ministry of Education of Brazil, Brasília - DF 70040-020, Brazil.

The seasonal fluctuations in zooplankton densities in temperate climates have been long known and the multiplicity of performed studies identified a vast number of factors responsible for these phenomena, such as changes in the physico-chemical factors and other such types of environmental forces governing them [1]. Acartia clausi is a euryaline temperate-boreal species very common in the Portuguese coastal ecosystems, in both estuarine and coastal waters [2]. It is usually described as a temperate water species of neritic calanoid copepod, which is associated with warmer water regions, and as a result becomes more abundant in the summer months, reaching a biomass maximum during the months of July and August. Growth and egg production have been studied extensively in some Acartia species $[1,2,3]$. In the present study histology and histochemistry were selected to determine the reproductive potential of $A$. clausi.

The ovigerous females were identified with a binocular microscope, isolated and fixed in the Bouin's solution for histological (5 $\mu \mathrm{m}$ thickness, mounting and Haematoxylin - Eosin staining) and histochemical analysis (Periodic Acid Schiff method (PAS) with Haematoxylin as a counter stain for the identification of the carbohydrate content and vitellogenic oocytes. The size of the oocytes was evaluated through measurements made with a micrometer.

The microscopic studies and Image analysis indicated that, in the month of September, the majority of the oocytes were immature and had reduced or almost negligible carbohydrate contents with very few vitellogenic oocytes (Figure $1 \mathrm{~A}$ and $\mathrm{B}$ ), while the specimens from the month of March exhibited a large difference in the oocyte dimensions. These were mature and more vitellogenic and occupied almost half the volume of the body (Figure $1 \mathrm{C}, \mathrm{D}$ and $\mathrm{E}$ ). This shows that, during the month of September, the environmental conditions are not favorable for the maturation of gonads in these species while in the month of March they proliferate and the species shows a high degree of reproductive potential.

This work forms a valid approach in understanding the population fluctuations and reproductive status in a key species of copepod showing a particular temporal variation associated with its reproductive strategy.

\section{References}

1. Lee W.Y. and McAlice B.J., Estuaries, 2:228-235, 1979.

2. Uye S., J. Exp. Mar. Biol. and Ecol., 50:255-271, 1981.

3. Giesel J.T., Annu. Rev. Ecol. Syst., 7:57-59, 1976. 

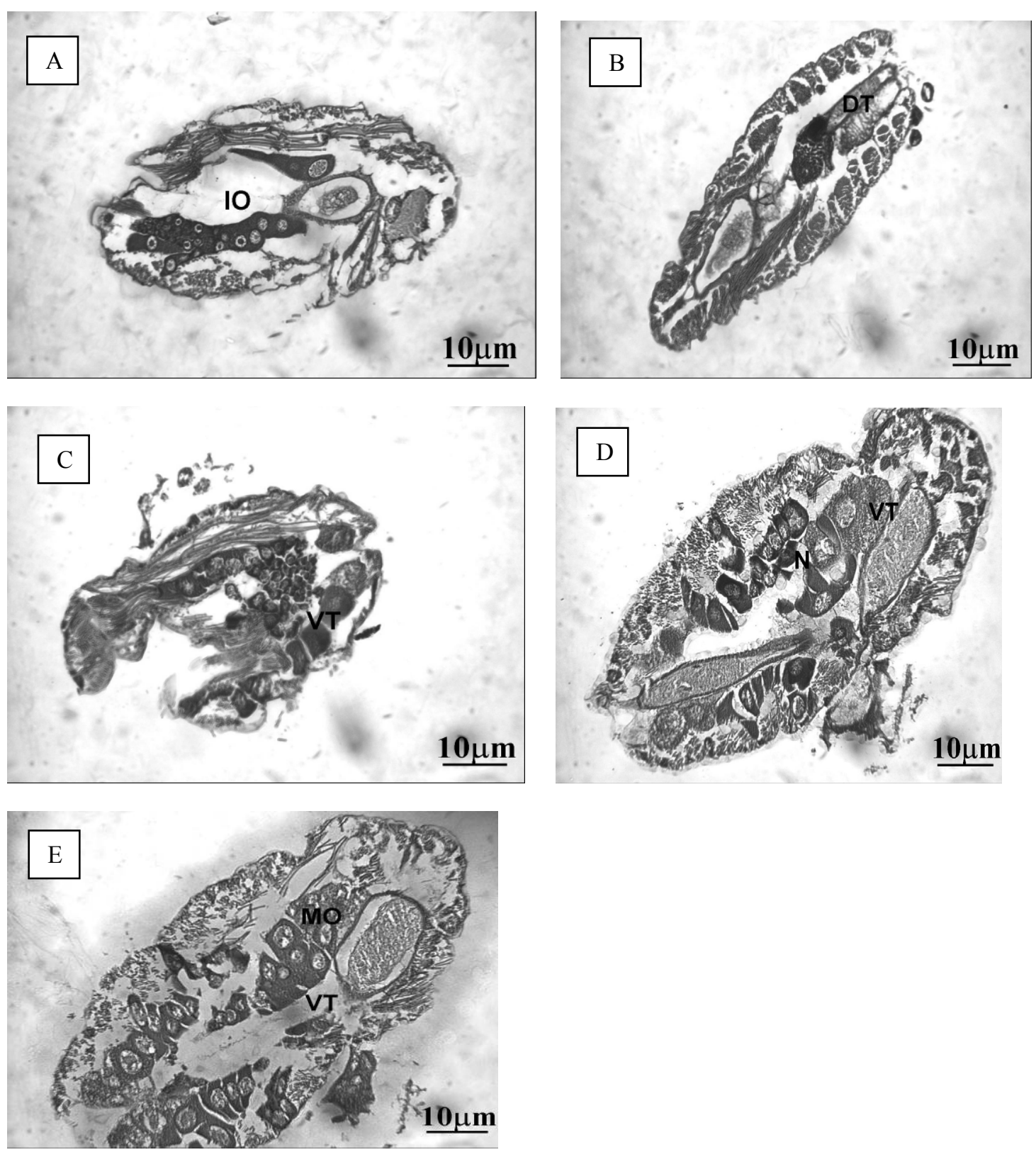

Figure 1. (A) Longitudinal section of $A$. clausi (Female), showing immature oocytes (IO). HE; (B) Longitudinal section of $A$. clausi (Female), showing the digestive tract and absence of gonads. HE; (C) Longitudinal section of A. clausi (Female), showing vitellogenic oocytes (VO). PAS; (D) Longitudinal section of A. clausi (Female), showing the nucleus (N) and vitellogenic oocytes (VO). PAS; (E) Longitudinal section of A. clausi (Female), showing the mature oocytes (MO) and vitellogenic oocytes (VO). PAS. 